\title{
Marcadores químicos em espécies de Echinodorus (Alismataceae) utilizadas como chapéu-de-couro
}

Chemical markers in species of Echinodorus (Alismataceae) used as "chapéu-de-couro"

\author{
R. R. Santos ${ }^{1 *}$; F. A. S. Fonseca1; A. M. Azevedo ${ }^{2}$; E. R. Martins ${ }^{1}$ \\ ${ }^{1}$ Instituto de Ciências Agrárias/Laboratório de Plantas Medicinais e Aromáticas/Universidade Federal de Minas \\ Gerais, 39404-547, Montes Claros-Minas Gerais, Brasil \\ ${ }^{2}$ Instituto de Ciências Agrárias/Laboratório de Experimentação Agrícola/Universidade Federal de Minas Gerais, \\ 39404-547, Montes Claros-Minas Gerais, Brasil \\ *riziarodrigues91@yahoo.com.br \\ (Recebido em 27 de novembro de 2020; aceito em 30 de abril de 2021)
}

\begin{abstract}
O nome chapéu-de-couro é dado às plantas pertencentes ao gênero Echinodorus (Alismataceae). São espécies nativas e popularmente utilizadas como diuréticas e anti-inflamatórias. As espécies Echinodorus macrophyllus e Echinodorus grandiflorus têm o uso recomendado pela Farmacopeia Brasileira. Entretanto, devido à plasticidade fenotípica do gênero, a identificação precisa é dificultada, consequentemente, Echinodorus floribundus e Echinodorus subalatus têm sido utilizadas pela população para os mesmos fins. O objetivo foi avaliar marcadores químicos para as espécies Echinodorus floribundus e Echinodorus subalatus e a similaridade química dessas com as espécies descritas na Farmacopeia Brasileira. A triagem fitoquímica revelou presença de fenóis, flavonoides, flavonas, flavonóis, xantonas, esteroides e triterpenoides nos extratos. Porém ambas as espécies apresentaram valores inferiores de flavonoides totais quando comparadas com Echinodorus scaber, descrita na literatura. A caracterização do extrato aquoso foliar em cromatografia líquida de alta eficiência (CLAE-DAD) detectou 118 e 121 compostos em E. floribundus e $E$. subalatus, respectivamente. Destes, 25 apresentaram espectros UV-Vis característicos de compostos fenólicos. Não foram detectados o ácido chicórico e a isoorientina, marcadores comuns em Echinodorus. Os teores de derivados do ácido o-hidroxicinâmico para E. floribundus e E. subalatus (5,52\% e 3,07\%, respectivamente) foram superiores ao que é exigido pela Farmacopeia Brasileira. Dessa forma, há diferenças químicas entre Echinodorus floribundus e Echinodorus subalatus e as descritas pela Farmacopeia Brasileira, entretanto, há indícios de potencial farmacológico das espécies estudadas.
\end{abstract}

Palavras-chave: flavonoides, fenóis, ácido $o$-hidroxicinâmico.

The name "chapéu-de-couro" is given to plants belonging to genus Echinodorus (Alismataceae) These are native and popularly used as diuretic and anti-inflammatory. The species E. macrophyllus and E. grandiflorus have the recommended use by the Brazilian Pharmacopeia. However, due to the phenotypic plasticity of the genus, precise identification is difficult, therefore, E. floribundus and E. subalatus have been used by the population for the same purpose. The aim of the work was to evaluate chemical markers for the species $E$. floribundus and E. subalatus and their chemical similarity with the species described in the Brazilian Pharmacopeia. Phytochemical screening revealed the presence of phenols, flavonoids, flavones, flavonols, xanthones, steroids and triterpenoids in the extracts. However, both species presented lower values of flavonoids and total phenols when compared with E. scaber. The characterization of aqueous leaf extract in high performance liquid chromatography (HPLC-DAD) detected 118 and 121 compounds in E. floribundus and E. subalatus, respectively. Of these, 25 showed characteristic UV-Vis spectra of phenolic compounds. Markers chicory acid and isoorientin, present in Echinodorus, weren't detected. The levels of $o$ hydroxycinnamic acid derivatives for E. floribundus and E. subalatus (5.52\% and 3.07\%, respectively) were higher than required by the Brazilian Pharmacopeia. Thus, there are chemical differences between Echinodorus floribundus and Echinodorus subalatus and those described by the Brazilian Pharmacopeia, however, there are indications of pharmacological potential of the species studied.

Keywords: flavonoids, phenols, $o$-hydroxycinnamic acid.

\section{INTRODUÇÃO}

O chapéu-de-couro, nome popular que denomina diversas espécies do gênero Echinodorus (Alismataceae), é predominantemente encontrado na região tropical da América do Sul $[1,2]$. 
Presente nas regiões Nordeste e Centro-Oeste do Brasil, tem como habitat lagoas temporais, depressões úmidas, pântanos, savanas e planícies inundadas [3, 4, 5, 6].

O extrato foliar do chapéu-de-couro, desde a década de 1940, é usado como matéria prima para preparo de refrigerante, associado à erva-mate, conferindo a bebida paladar agradável [7, 8]. A adição do mesmo à cerveja ale também é alvo de estudo, visando obtenção de bebida com características sensoriais diferenciadas e elevada atividade antioxidante [9].

O chapéu-de-couro é importante para o paisagismo, várias espécies pertencentes são importadas para países da Europa com essa finalidade [10]. Também, pode ser utilizado na descontaminação de aquíferos poluídos [11, 12], uma vez que este demonstra-se eficiente na fitorremediação de chumbo a níveis de traços [13-15].

As folhas de Echinodorus macrophyllus e Echinodorus grandiflorus são utilizadas na medicina popular [16], no tratamento de doenças do sistema gastrointestinal e de doenças relacionadas com obstetrícia, ginecologia e trato urinário [17]. De acordo com Dias et al. (2013) [18], a espécie $E$. macrophyllus teve o seu uso recomendado na Farmacopeia Brasileira $1^{a}$ edição, de 1926, e na Farmacopeia Brasileira 2a edição, de 1959. Já a espécie E. grandiflorus foi introduzida na Farmacopeia Brasileira $5^{\text {a }}$ edição (2010) [19], e se manteve na Farmacopeia Brasileira $6^{\text {a }}$ edição (2019), como a espécie da qual é obtida a droga vegetal do chapéu-de-couro [20]. Para ambas, foram confirmadas atividades biológicas com propriedades diurética, antinecrótica, tumoral, antiinflamatória e capacidade de interromper ou pelo menos retardar lesões renais [16, 21-24].

Conseguinte, compostos como diterpenos, alcaloides, glicosídeos, saponinas, flavonoides, ácidos fenólicos e derivados do ácido $o$-hidroxicinâmico [18, 25-27] foram isolados nas espécies E. macrophyllus e E. grandiflorus. A variação dos principais compostos detectados em Echinodorus pode ser atribuída às diferentes técnicas de extração utilizadas, à capacidade de adsorção e à volatilidade dos compostos [28]. Sugere-se que os flavonoides são responsáveis pelas atividades biológicas comprovadas, em sinergia ou não com outros compostos [7, 22, 24, 29, 30]. Dentre os quais, destacam-se: swertisin, orientina, isoorientina, swertiajaponine, vitexina e isovitexina, além dos ácidos trans aconítico e chicórico [7, 22, 24, 29, 30]. Contudo, é desconhecido o perfil químico das espécies Echinodorus floribundus (Seub.) Seub. e Echinodorus subalatus (Mart.) Griseb., utilizadas como chapéu-de-couro no Norte e Vale do Jequitinhonha, em Minas Gerais, Brasil.

Também, a dificuldade de identificação precisa das espécies, apresenta-se como um dos maiores problemas para o gênero Echinodorus [31]. Visto que as espécies apresentam plasticidade fenotípica, associada à baixa quantidade de estruturas morfológicas convergentes [31]. De modo que é comum encontrar na literatura autores que consideram a espécie E. floribundus como sinonímia a E. grandiflorus spp. aureus $[32,33]$ e autores que a classificam como espécie distinta $[3,31,34,35]$.

Frente ao exposto, a ampliação dos estudos químicos com as demais espécies utilizadas pela população faz-se necessária, a fim de garantir o uso seguro das mesmas, além de contribuir na obtenção de informações que auxiliem na identificação em nível de espécie e na produção de compostos vegetais biologicamente ativos de interesse da indústria de fitofármacos, o que pode cooperar no desenvolvimento de estudos de manejo, domesticação e conservação dessas espécies. O objetivo foi obter marcadores químicos para as espécies Echinodorus floribundus e Echinodorus subalatus e avaliar a similaridade química entre as espécies estudadas com as espécies descritas pela Farmacopeia Brasileira.

\section{MATERIAL E MÉTODOS}

\subsection{Coleta do material vegetal}

Para a realização da coleta do material vegetal em campo, foi concedida pelo Instituto Estadual de Florestas (IEF), a autorização para pesquisa científica no estado de Minas Gerais, ${ }^{\circ}$ 041/2018, cadastro no SisGen ${ }^{\circ}$ A97D542. Foram realizadas consultas públicas ao Herbário Virtual Reflora para identificação dos 15 pontos de coleta por meio do levantamento de dados e coordenadas de exsicatas registradas como Echinodorus spp., localizadas nas regiões Norte e Vale do 
Jequitinhonha, MG. As coletas ocorreram entre os meses de agosto de 2016 a janeiro de 2017, época chuvosa nas regiões de estudo. As amostras foram coletadas e armazenadas em sacos plásticos. Materiais floridos foram prensados, secos e encaminhados para identificação com auxílio da chave botânica do gênero [36], realizada pela Profa. Rúbia Santos Fonseca, curadora do Herbário MCCA do Instituto de Ciências Agrárias da Universidade Federal de Minas Gerais (ICA/ UFMG), sendo depositadas as exsicatas MCCA 2595 e MCCA 3123. Nove acessos foram identificados da espécie Echinodorus floribundus e seis acessos Echinodorus subalatus, totalizando 15 acessos de ambas as espécies (Figura 1).

Dessas plantas foi estabelecida coleção de germoplasma em casa de vegetação, no ICA/ UFMG $\left(16^{\circ} 40^{\prime} 58,5^{\prime \prime} \mathrm{S}, 43^{\circ} 50^{\prime} 25,6^{\prime \prime} \mathrm{L}\right)$ e altitude de 626 metros. O clima da região é caracterizado como tropical típico, quente e semiúmido, com estação chuvosa curta (verão) e seca prolongada (inverno), bem definida [37]. A coleção de germoplasma foi formada com o plantio dos indivíduos de cada acesso em vasos de $5 \mathrm{dm}^{3}$, utilizado como substrato a mistura solo:areia:esterco na proporção 3:2:1. A irrigação foi feita manualmente, com saturação do solo e intervalos de dois dias. Após o estabelecimento da coleção de germoplasma, deu-se início as análises químicas. A fim de ser utilizadas como padrão de comparação, amostras comerciais de chapéu-de-couro, identificadas pelo produtor como Echinodorus macrophyllus foram adquiridas no comércio de Montes Claros - MG.

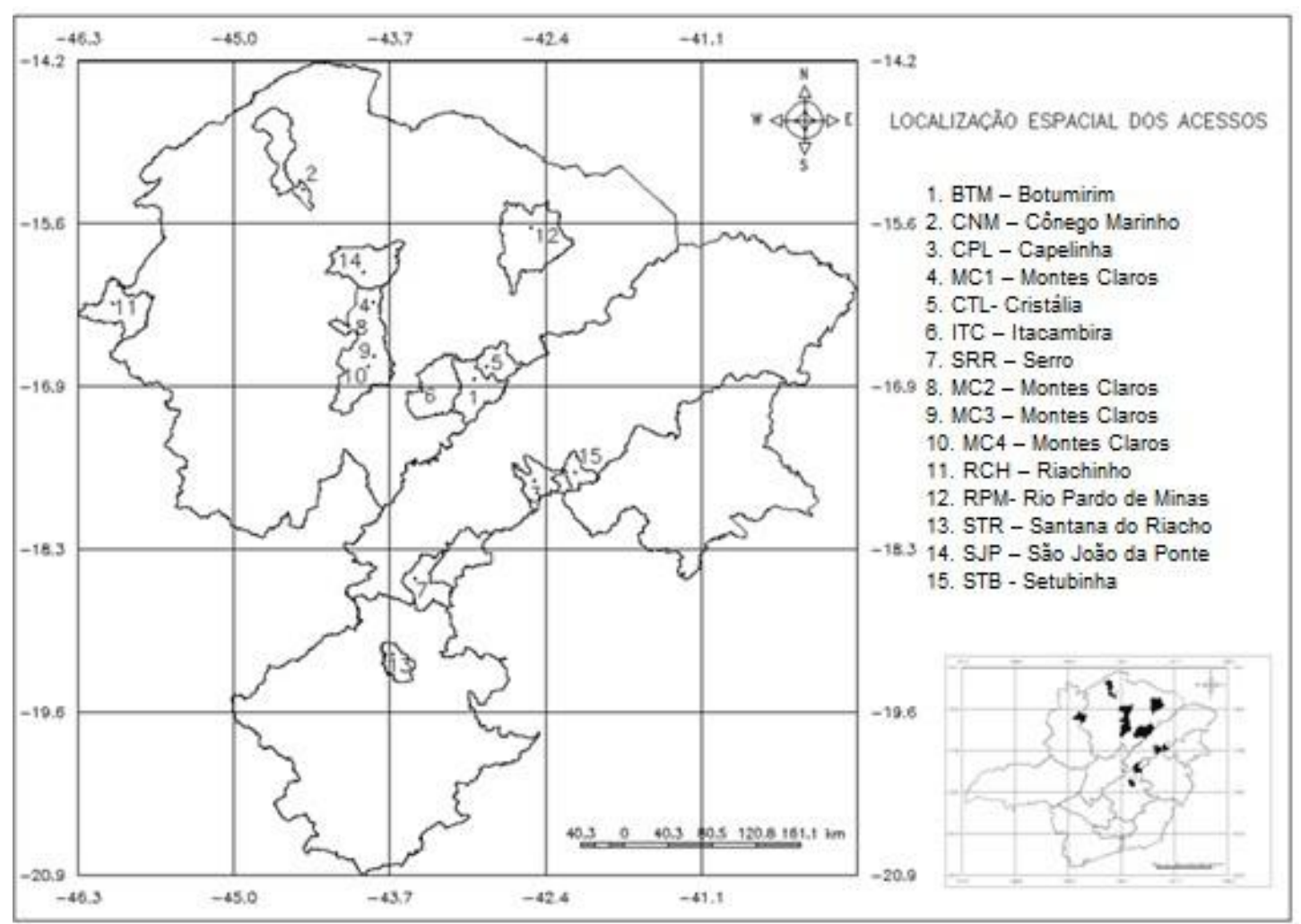

Figura 1: Carta política das mesorregiões Norte, Vale do Jequitinhonha, Vale do Mucuri e Metropolitana de Belo Horizonte, em Minas Gerais, Brasil, com pontos representando os 15 acessos de ocorrência de Echinodorus floribundus $e$ Echinodorus subalatus [38].

\subsection{Triagem fitoquímica}

Para o preparo dos extratos, do banco de germoplasma foram coletadas amostras com aproximadamente $3,24 \mathrm{~g}$ de folhas frescas de E. floribundus e $103,16 \mathrm{~g}$ de folhas frescas de $E$. subalatus, com umidade média de $67 \%$. O preparo dos extratos aquosos das espécies de $E$. floribundus e E. subalatus foi realizado a partir de folhas frescas $(0,36 \mathrm{~g})$ pulverizadas com auxílio do nitrogênio líquido, adicionada água destilada $(20 \mathrm{~mL})$ e levados a banho-maria $98{ }^{\circ} \mathrm{C}(15$ minutos). Após atingir temperatura ambiente, a mistura foi filtrada com papel filtro (26 $\mu \mathrm{m})$ e os 
extratos foram armazenados em frascos âmbar, na concentração final de $18 \mathrm{mgmL}^{-1}$. Os testes de detecção utilizados para esses compostos foram com cloreto férrico e hidróxido de sódio [39]. Alíquotas dos extratos aquosos foram transferidas para tubos de ensaio $(3 \mathrm{~mL})$, aos quais se acrescentaram cinco gotas de cloreto férrico a $2 \%$ e deixados em repouso sob temperatura ambiente por cerca de três minutos. Alíquotas dos extratos aquosos foram transferidas para tubos de ensaio (3 mL), aos quais foi acrescentada solução aquosa de hidróxido de sódio a 10\% (1 mL) e, posteriormente, foram agitados.

O teste com o cloreto férrico, descrito anteriormente, também foi utilizado na detecção de taninos [39]. Além desse, foram realizadas as reações com acetato neutro de chumbo e com solução de acetato decobre [39]. Uma alíquota dos extratos aquosos (1 mL) foi diluída na proporção 1:5 e transferida para tubos de ensaio, aos quais foram adicionadas duas gotas de acetato neutro de chumbo a $10 \%$ em repouso sob temperatura ambiente. Outra alíquota dos extratos aquosos $(1 \mathrm{~mL})$ foi diluída na proporção 1:5, transferida para tubos de ensaio e adicionadas duas gotas de solução aquosa de acetato de cobre em repouso, sob temperatura ambiente. Para análise de saponinas, foi preparado extrato com $1 \mathrm{~g}$ de folhas frescas pulverizadas com auxílio do nitrogênio líquido, e 100 $\mathrm{mL}$ de água destilada, em ebulição por um minuto. Posteriormente, o extrato foi filtrado em papel filtro e separado para teste qualitativo de espuma [40]. Os testes para detecção de antocianinas, antocianidinas, flavonóis, flavanonas, flavanonóis, xantonas, esteroides e triterpenos foram realizados com extrato hidroetanólico preparado da seguinte maneira: $100 \mathrm{~g}$ de folhas frescas foram pulverizadas com auxílio de nitrogênio líquido, acrescentou-se $500 \mathrm{~mL}$ de solução hidroetanólica $70 \%$, para maceração exaustiva por sete dias, à temperatura ambiente, sob agitação diária, após esse período, a mistura foi aquecida em banho-maria e filtrada em papel de filtro. Para ajustar a concentração para 2:1 (planta:solvente), o extrato foi levado ao evaporador rotativo, à pressão de $200 \mathrm{mmHg}$ e $85^{\circ} \mathrm{C}$.

\subsection{Fenóis e flavonoides totais}

Para o preparo dos extratos metanólicos utilizados nas análises foram coletadas folhas frescas de cada acesso e pulverizadas com auxílio de nitrogênio líquido. Foi adicionado metanol $(25 \mathrm{~mL})$ sobre as folhas pulverizadas $(0,36 \mathrm{~g})$ e levada a mistura ao banho-maria $\left(98^{\circ} \mathrm{C}\right)$ por 30 minutos. Após atingir temperatura ambiente, foi realizada a filtragem dos extratos com papel filtro $(26 \mu \mathrm{m})$ em balão de $50 \mathrm{~mL}$, o resíduo foi lavado com $25 \mathrm{~mL}$ de metanol e o volume foi aferido até o menisco e armazenados em frascos âmbar, na concentração final de $7,2 \mathrm{mg} \mathrm{mL}^{-1}$.

A quantificação de fenóis totais foi realizada, segundo Amorin et al. (2008) [41], pelo método de Folin e Ciocalteu. A curva de calibração experimental foi preparada utilizando alíquotas de solução aquosa de ácido tânico $\left(5 \mathrm{~mL}, 0,1 \mathrm{mg} \mathrm{mL}^{-1}\right)$, de reagente Folin-Ciocalteu e de carbonato de sódio $(10 \mathrm{~mL}, 10,6 \% \mathrm{~m} / \mathrm{v})$, que resultaram em concentrações finais de 0,$1 ; 0,2 ; 0,3 ; 0,4$ e $0,5 \mu \mathrm{g}$ $\mathrm{mL}^{-1}$ de ácido tânico. Após $30 \mathrm{~min}$ de reação, a leitura da absorbância realizada em espectrofotômetro (Micronal B-582) a $760 \mathrm{~nm}$. Os extratos metanólicos de E. floribundus e $E$. subalatus $(1 \mathrm{~mL})$ foram diluídos em água (1:99) e submetidos ao mesmo procedimento. A equação da curva de calibração foi y $=0,081 \mathrm{x}+0,0025$, em que y é a absorbância (nm) e x a concentração $\left(\mu \mathrm{g} \mathrm{mL}^{-1}\right)$, expressa em equivalentes de ácido tânico por grama de folha.

A quantificação de flavonoides totais, seguiu a metodologia de Peixoto Sobrinho et al. (2008), com adaptação na concentração da solução de cloreto de alumínio [42]. A curva de calibração experimental foi preparada utilizando alíquotas de solução de rutina $\left(0,5 \mathrm{mg} \mathrm{mL}^{-1} \mathrm{em}\right.$ metanol), cloreto de alumínio $\left(2,5 \mathrm{~mL}, 500 \mathrm{mg} \mathrm{mL}^{-1} \mathrm{em}\right.$ metanol), resultando em concentrações finais de 20,$0 ; 34,0 ; 48,0 ; 62,0$ e 76,0 $\mu \mathrm{g} \mathrm{mL}^{-1}$ de rutina. A mistura reacional ficou em repouso por $30 \mathrm{~min} \mathrm{e}$ posteriormente a absorbância lida a $410 \mathrm{~nm}$. Os extratos metanólicos de E. floribundus e $E$. subalatus $(1 \mathrm{~mL})$ foram diluídos em metanol (1:24) e submetidos ao mesmo procedimento. A equação da curva de calibração foi $y=0,0072 x+0,4811$, em que y é a absorbância (nm) e x a concentração $\left(\mu \mathrm{g} \mathrm{mL}^{-1}\right)$, expressa posteriormente em equivalentes de rutina por grama de folha. 


\subsection{Análise cromatográfica do extrato foliar}

Foram coletadas folhas dos acessos de E. floribundus (BTM, CNM, CPL, CTL, ITC e STR) e E. subalatus (MC1, MC2, MC4 e SJP), separadas em pecíolos e limbos para higienização, as partes vegetais foram lavadas com água destilada. As folhas de cada acesso foram pesadas $(20 \mathrm{~g})$, transferidas para almofariz de porcelana e maceradas com nitrogênio líquido. O macerado foi submetido à infusão com água destilada fervente, até atingir a temperatura de ambiente $\left(23^{\circ} \mathrm{C}\right) . \mathrm{O}$ extrato foi filtrado em papel de filtro $(26 \mu \mathrm{m})$ e liofilizado durante 24 horas à temperatura de -10 ${ }^{\circ} \mathrm{C}$ e 2,5 mbar de pressão, em liofilizador modelo Alpha 1-2 LD plus da Christ.

Os extratos foram ressuspensos em água ultra pura, injetados na concentração de $10 \mathrm{mg} \mathrm{mL}^{-1}$ em cromatógrafo líquido de alta eficiência, modelo LC-20 da Shimadzu, com amostrador automático (SIL-20A) e detector de arranjo de diodos (SPD-M20A) controlado por software LCSolution 1.0. A coluna utilizada foi a C-18 $(250 \mathrm{~mm} \times 4 \mathrm{~mm}$ id, $5 \mu \mathrm{m})$ em combinação com précoluna C-18 (4 mm x $4 \mathrm{~mm}$ id, $5 \mu \mathrm{m}$ ) e o volume de injeção de $10 \mu \mathrm{L}$. As fases móveis utilizadas foram água ultrapura (A) e acetonitrila (B), cada uma contendo 0,01\% de ácido fosfórico (v/v). O gradiente iniciou com $95 \% \mathrm{~A}$, após 20 minutos $72 \% \mathrm{~A}$, aos 30 minutos $5 \% \mathrm{~A}$, aos 35 minutos a proporção utilizada foi de $95 \%$ A. O tempo total de análise foi de 35 minutos, com temperatura de

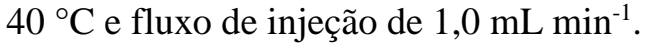

Foram preparadas e injetadas $10 \mu \mathrm{L}$ das soluções padrões de ácido chicórico (Sigma-Aldrich, $\geq$ 95\% de pureza, grau HPLC, $8 \mathrm{mg} \mathrm{mL}^{-1}$ em água ultrapura) e isoorientina (Sigma Aldrich, $\geq 98 \%$ de pureza, grau HPLC $0,5 \mathrm{mg} \mathrm{mL}^{-1} \mathrm{em}$ metanol grau HPLC). Os cromatogramas das amostras e dos padrões foram obtidos em espectros de UV na faixa de 195 a $400 \mathrm{~nm}$ e processados a $210 \mathrm{~nm}$ [43].

\subsection{Derivados do ácido o-hidroxicinâmico}

A determinação do teor de derivados do ácido o-hidroxinamico nas amostras de E. floribundus, E. subalatus e comercial foi realizada conforme adaptação da metodologia da Farmacopeia Brasileira, em triplicata (2019) [20].

\subsection{Análise estatística dos dados}

As análises estatísticas foram feitas em triplicata com o auxílio do software R [44].

\section{RESULTADOS E DISCUSSÃO}

\subsection{Triagem fitoquímica, flavonoides e fenóis totais}

Nos testes utilizando reativos específicos, as reações foram positivas para as classes fenóis e flavonoides, confirmados pela alteração da coloração dos extratos [38]. No teste de detecção de taninos não houve a formação de precipitado. Também, não houve formação de espuma no teste de saponinas, o que indica a ausência de taninos em ambas as espécies estudadas e ausência de saponinas em E. subalatus [38, 39]. Nos testes de deteç̧ão de antocianinas, antocianidinas, flavonóis, flavanonas, flavanonóis, xantonas, esteroides e triterpenos, nos quais se utilizou o extrato hidroetanólico de E. subalatus, foram observados resultados positivos para flavonas, flavonóis, flavanonóis e xantonas; esteroides e triterpenoides, de acordo com a metodologia adotada [38]. Tanus-Rangel et al. (2010) [29], ao estudarem atividade biológica do extrato etanólico das folhas de Echinodorus macrophyllus, atribuíram o efeito anti-inflamatório à presença de triterpenos, esteroides, flavonas, xantonas e flavonoides. Os flavonoides também são alvos de estudo do extrato hidroetanólico das folhas de Echinodorus grandiflorus, sendo estes responsáveis pela atividade anti-inflamatória e diurética [22, 23]. Os demais grupos funcionais, antocianinas e antocianidinas, não foram detectados pelos testes realizados. Umas das possíveis causas seria a solução extratora utilizada. Por serem compostos de caráter polar, suas extrações são recomendadas utilizando 
soluções alcoólicas a base de metanol ou etanol acidificado [45]. Porém, no presente estudo foi utilizada solução hidroetanólica ao pH 3 e alcalinizadas a pH 8,5 e 11, mesmo assim, não foram observados resultados positivos, o que pode indicar baixa concentração desses compostos, ou até mesmo ausência dos compostos em E. subalatus.

$\mathrm{Na}$ literatura, é possível observar falta de padronização dos métodos de quantificação espectrofotométrica de flavonoides e fenóis totais para as espécies de Echinodorus. Ocorrem variações nos padrões de equivalência e unidades de quantificação $\left(\mu \mathrm{g} \mathrm{mL}^{-1}\right.$ até $\mathrm{g} / \mathrm{g}$ de extrato) [24, $30,43,46,47]$. A rutina foi o padrão utilizado na quantificação totatal de flavonoides, e o ácido tânico na quantificação total de fenóis. Para fins de comparação, os resultados da literatura para flavonoides e fenóis totais, bem como os do estudo foram convertidos em mg equivalentes de padrão por grama de folha (Tabela 1).

Tabela 1: Teor de flavonoides e fenóis totais em amostras de chapéu-de-couro (Echinodorus sp.).

\begin{tabular}{|c|c|c|c|}
\hline \multirow[b]{2}{*}{ Espécie } & \multicolumn{2}{|c|}{ Experimento } & \multirow{2}{*}{ Literatura } \\
\hline & Flavonoidestotais & Fenóis totais & \\
\hline E. floribundus & $\begin{array}{l}2,80-5,00 \pm 0,65 \mathrm{mg} \\
\text { equivalentes de rutina/ } \mathrm{g} \text { de } \\
\text { folha }\end{array}$ & $\begin{array}{l}0,40-5,30 \pm 2,01 \mathrm{mg} \\
\text { equivalentes de ácido } \\
\text { tânico/g de folha }\end{array}$ & - \\
\hline E. subalatus & $\begin{array}{l}3,50 \pm 0,25 \mathrm{mg} \text { equivalentes de } \\
\text { rutina/g de folha }\end{array}$ & $\begin{array}{l}1,63 \quad \pm \quad 0,54 \quad \mathrm{mg} \\
\text { equivalentes de } \\
\text { tânico/g de folha }\end{array}$ & - \\
\hline $\begin{array}{l}\text { Amostra } \\
\text { comercial }\end{array}$ & $\begin{array}{l}0,01 \pm 0,0005 \mathrm{mg} \text { equivalentes } \\
\text { de rutina/ } \mathrm{g} \\
\text { de folha }\end{array}$ & $\begin{array}{l}0,09 \quad \pm \quad 0,01 \quad \mathrm{mg} \\
\text { equivalentes } \mathrm{de} \text { ácido } \\
\text { tânico/ g de folha }\end{array}$ & - \\
\hline \multirow[t]{2}{*}{ E. grandiflorus } & $\begin{array}{l}8225 \mathrm{mg} \text { equivalentes de } \\
\text { rutina/g de folha }\end{array}$ & - & [43] \\
\hline & $\begin{array}{l}98,13 \text { a } 363,75 \mathrm{mg} \text { equivalentes } \\
\text { de vitexina/ } \mathrm{g} \text { folha }\end{array}$ & $\begin{array}{l}7,08 \mathrm{mg} \text { equivalentes de } \\
\text { catequina/ } \mathrm{g} \text { de folha }\end{array}$ & {$[30]$} \\
\hline \multirow[t]{2}{*}{ E. macrophyllus } & $\begin{array}{l}2,90 \mathrm{mg} \text { equivalentes de } \\
\text { quercetina/ } \mathrm{g} \text { de folha }\end{array}$ & - & [46] \\
\hline & $\begin{array}{l}33,50 \mathrm{mg} \text { equivalente de } \\
\text { quercetina/ } \mathrm{g} \text { de folha }\end{array}$ & - & {$[24]$} \\
\hline \multirow[t]{2}{*}{ E. scaber } & $\begin{array}{l}197,78 \mathrm{mg} \text { equivalentes de } \\
\text { rutina/g de folha }\end{array}$ & $\begin{array}{l}222,20 \mathrm{mg} \text { equivalentes } \\
\text { de ácido gálico/ } \mathrm{g} \text { de folha }\end{array}$ & {$[47]$} \\
\hline & $\begin{array}{l}7,50 \text { a } 584,38 \mathrm{mg} \text { equivalentes } \\
\text { de vitexina/ } \mathrm{g} \text { de folha }\end{array}$ & $\begin{array}{l}8,25 \mathrm{mg} \text { equivalentes de } \\
\text { catequina/ } \mathrm{g} \text { de folha }\end{array}$ & {$[30]$} \\
\hline
\end{tabular}

Quanto ao conteúdo total de flavonoides, em E. floribundus houve variação de 2,80 a 5,00 mg equivalentes de rutina/ $\mathrm{g}$ de folha e, em E. Subalatus, a média de flavonoides totais foi de 3,50 $\mathrm{mg}$ equivalentes de rutina/ $\mathrm{g}$ de folha. Para a espécie $E$. floribundus foi possível observar variação de 0,40 a 5,03 mg equivalentes de ácido tânico/ g de folha no conteúdo total de fenóis, já para $E$. subalatus, a quantidade média de fenóis foi de $1,63 \mathrm{mg}$ equivalentes de ácido tânico/ $\mathrm{g}$ de folha (Tabela 1). Esses valores foram menores do que os relatados por Rosa et al. (2017) [47] para $E$. scaber, que adotou mesma metodologia de quantificação utilizada neste estudo (Tabela 1). As amostras comerciais obtiveram os menores valores de fenóis e flavonoides totais (Tabela 1).

\subsection{Perfil do extrato por CLAE-DAD}

As análises cromatográficas detectaram a presença de 118 compostos nos acessos de $E$. floribundus. Desse total, 113 compostos foram observados em todos os acessos. Três compostos 
foram encontrados apenas no acesso Santana do Riacho (STR). O acesso Itacambira (ITC) não apresentou dois compostos que foram detectados nos demais acessos. Para a espécie E. subalatus, foram detectados 121 compostos, dos quais apenas 115 desses foram observados em todos os acessos. Os acessos Montes Claros 1 (MC1) e São João da Ponte (SJP) apresentaram três compostos que não foram detectados em nenhum dos outros acessos da espécie. Já o acesso Montes Claros 2 (MC2) registrou ausência de um composto comum aos demais acessos. Contudo, nenhum dos compostos foi possível ser identificado devido ao custo elevado dos padrões. Dentre todos os compostos detectados nas duas espécies, 25 se destacaram por apresentarem espectro UV-Vis característicos de compostos fenólicos (Figuras 2 e 4), com absorção máxima nas bandas 240 a 280 nm e 300 a 380, características de flavonoides (isoflavonas, flavonas, flavonóis e chalconas) [48].
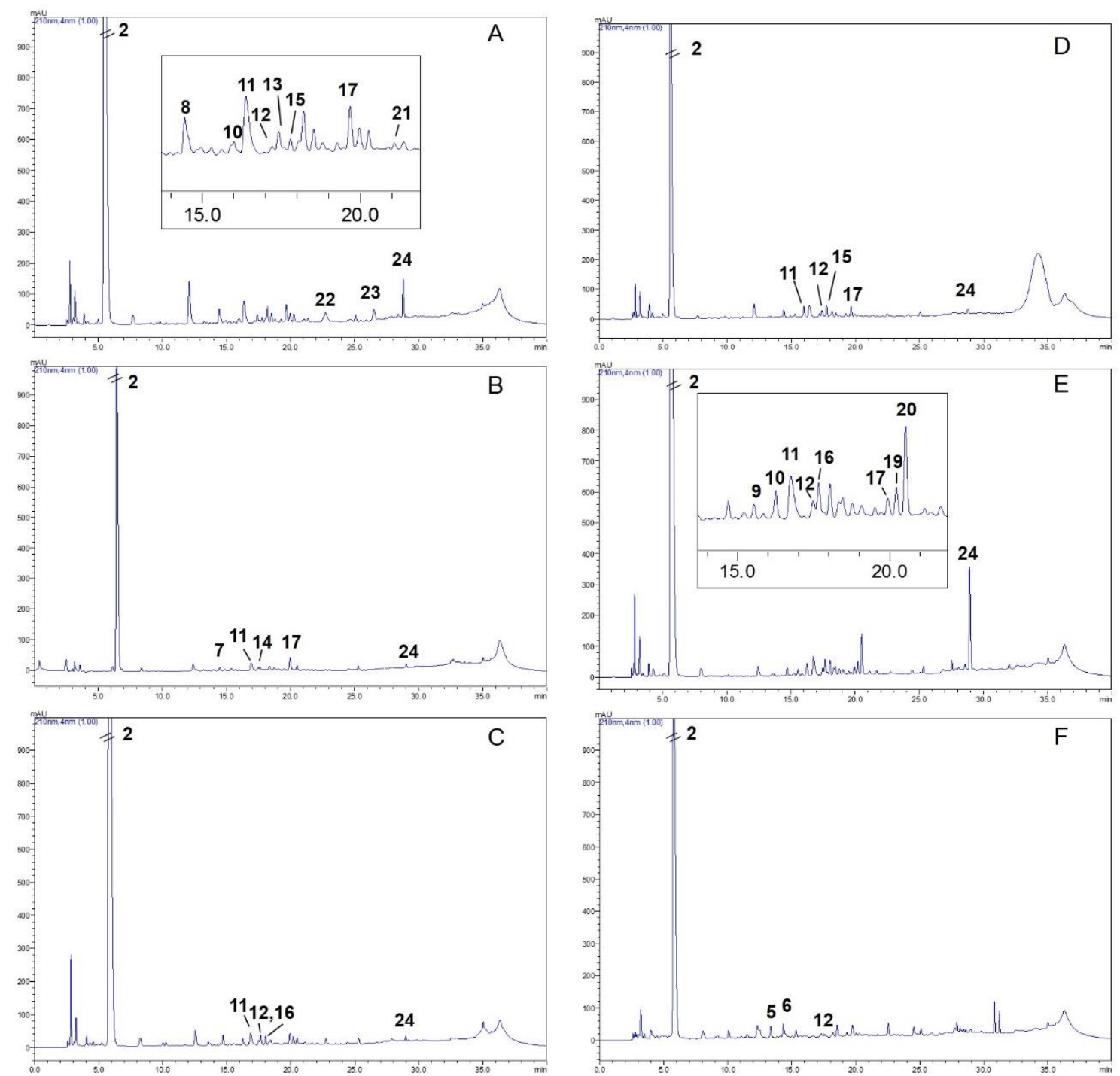

Figura 2: Cromatogramas dos extratos aquosos das folhas de Echinodorus floribundus (A-F) analisados por CLAE-DAD. (1-25) compostos que apresentam espectro UV-Vis característicos de fenólicos; (A) BTM:

Botumirim; (B) CNM: Cônego Marinho; (C) CPL: Capelinha; (D) CTL: Cristália; (E) ITC: Itacambira;

(F) STR: Santana do Riacho.

Desses, dois compostos, de número 2 e 24 , se destacam pela ocorrência frequente nos acessos das duas espécies (Figuras 3 e 5, Tabela 2), 15 compostos foram exclusivamente em acessos de $E$. floribundus (Tabela 2) e quatro compostos em acessos de E. subalatus (Tabela 2). Em nenhum dos acessos das duas espécies estudadas foi possível detectar o flavonoide isoorientina e o ácido chicórico, que são compostos descritos para as espécies E. grandiflorus e E. macrophyllus. 
1)
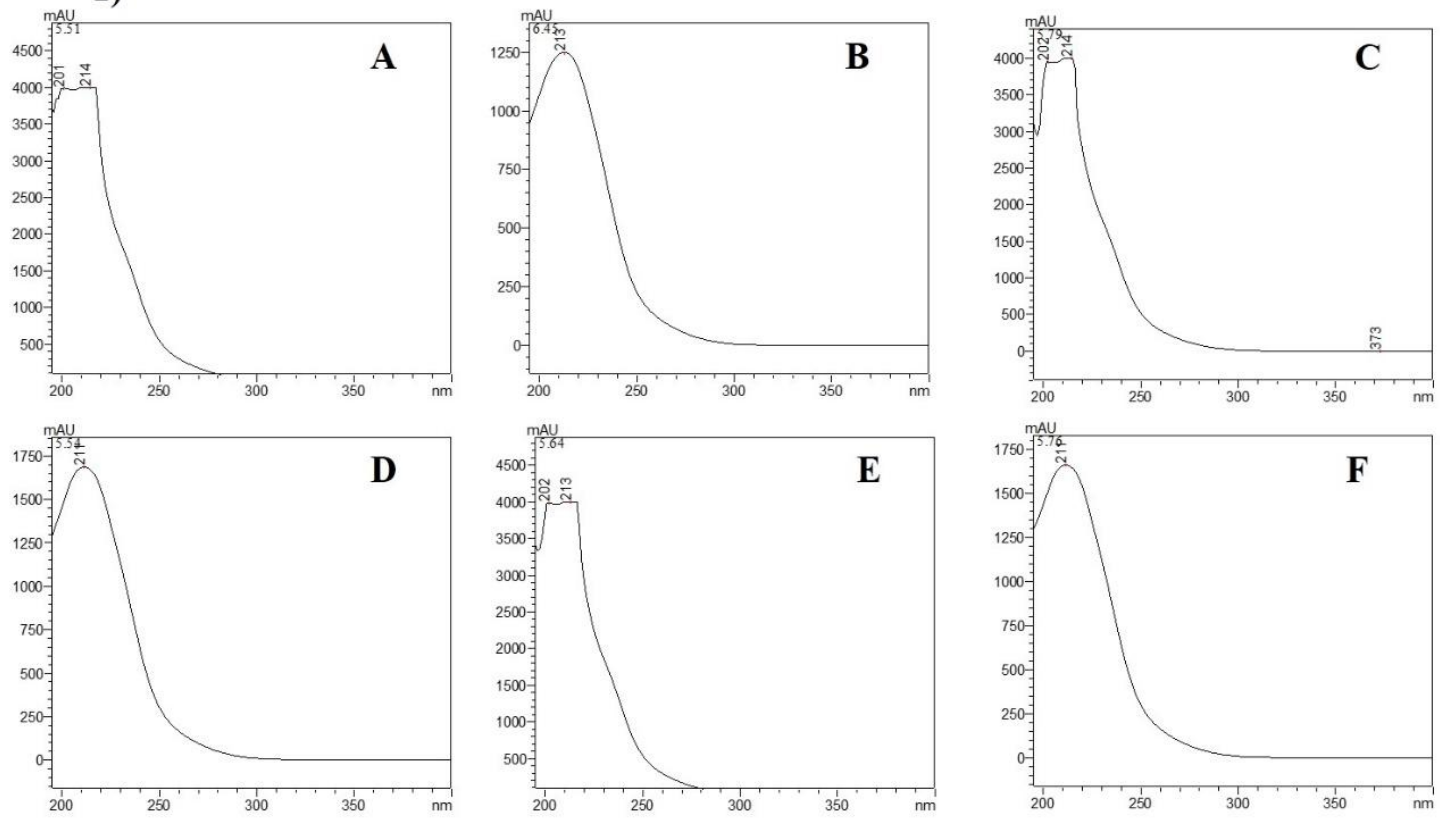

2)
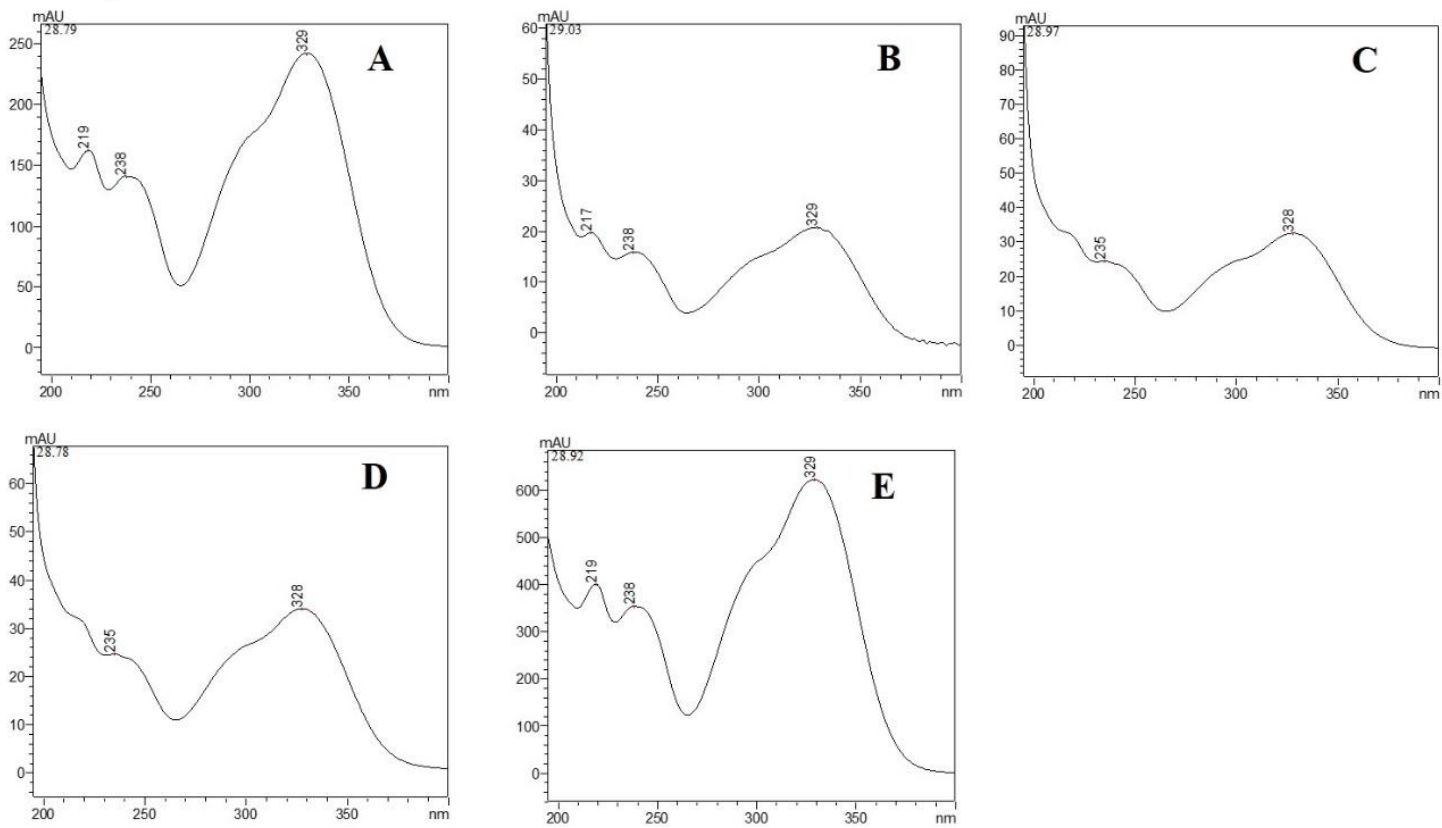

Figura 3: Espectros UV-Vis dos compostos 2 (1) e 24 (2), característicos de fenólicos, dos extratos aquosos das folhas de Echinodorus floribundus (A-F) analisados por CLAE-DAD; (A) BTM: Botumirim;

(B) CNM: Cônego Marinho; (C) CPL: Capelinha; (D) CTL: Cristália; (E) ITC: Itacambira; (F) STR: Santana do Riacho. 

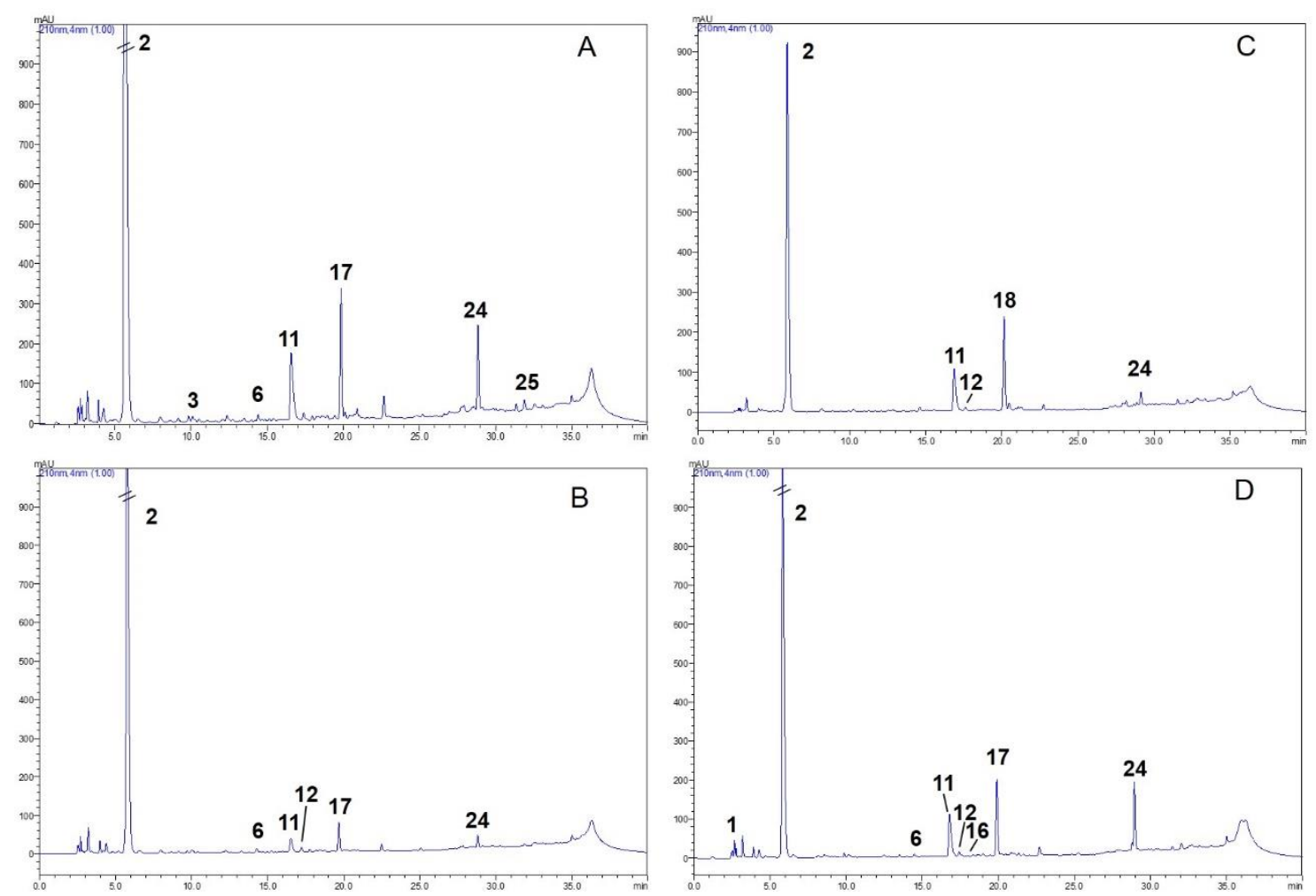

Figura 4: Cromatogramas dos extratos aquosos das folhas de Echinodorus subalatus (A-D) analisados por CLAE-DAD. (1-25) compostos que apresentam espectro UV-Vis característicos de fenólicos;(A) MCl: Montes Claros 1; (B) MC2: Montes Claros 2; (C) MC4: Montes Claros 4; (D) SJP: São João da Ponte. 
1)
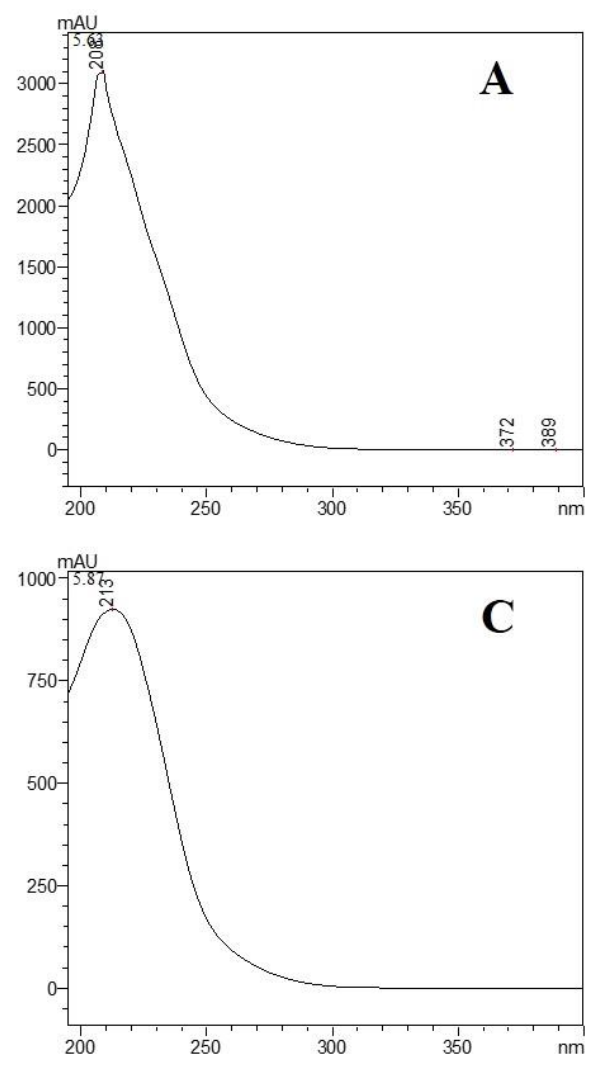

2)
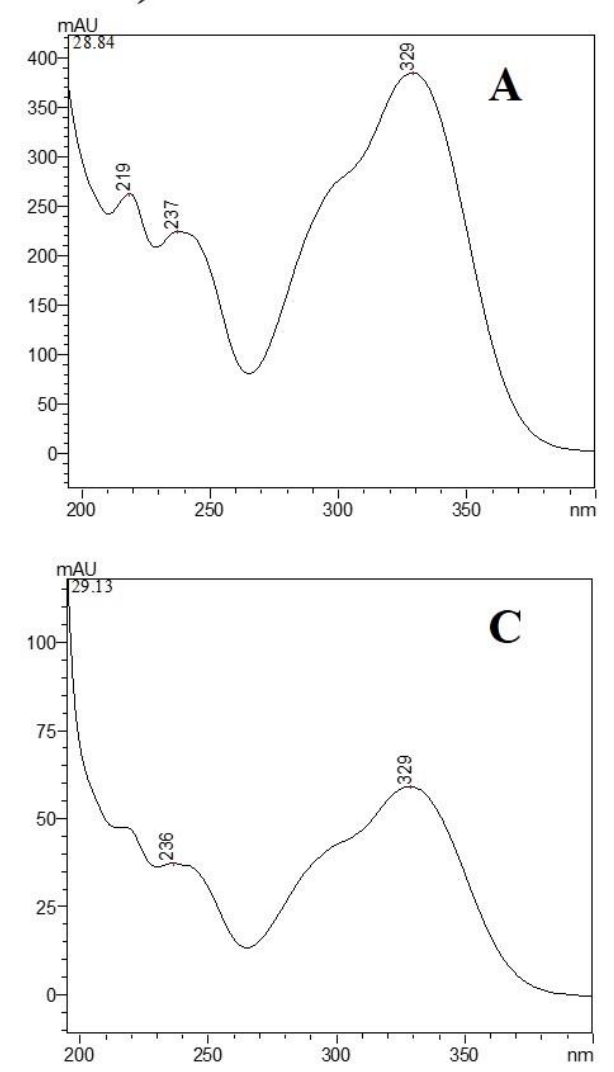
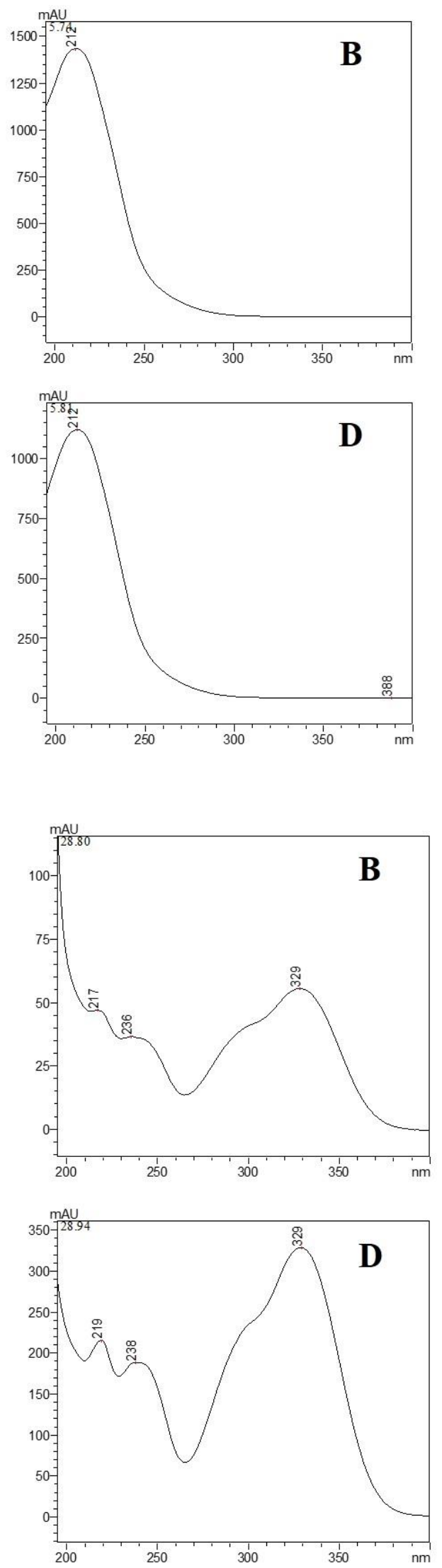

Figura 5: Espectros UV-Vis dos compostos 2 (1) e 24 (2), característicos de fenólicos obtidos em análise por CLAE-DAD dos extratos aquosos das folhas de Echinodorus subalatus (A-D); (A) MC1: Montes Claros 1; (B) MC2: Montes Claros 2; (C) MC4: Montes Claros 4; (D) SJP: São João da Ponte. 
Tabela 2: Compostos detectados em CLAE em extratos foliares de Echinodorus floribundus e Echinodorus subalatus com características de compostos fenólicos.

\begin{tabular}{|c|c|c|c|}
\hline Composto & Espécie & TR (min) & $\lambda(\mathbf{n m})$ \\
\hline 1 & E. subalatus (SJP) & 2,5 & $202 ; 212 ; 278 ; 361$ \\
\hline 2 & E. floribundus (BTM, CNM, CPL, CTL, ITC, SDC), E. subalatus (MC1, MC2, MC4, SJP) & $5,5-6,4$ & $201 ; \mathbf{2 0 2} ; 208 ; \mathbf{2 1 1} ; \mathbf{2 1 3} ; \mathbf{2 1 4} ; \mathbf{3 6 9} ; 372 ; 374$ \\
\hline 4 & E. floribundus (BTM) & 12,1 & $207 ; 325$ \\
\hline 5 & E. floribundus (SDC) & 13,3 & $220 ; 265$ \\
\hline 6 & E. floribundus (SDC), E. subalatus (MC1, MC2, SJP) & $14,0-14,4$ & 204; $205 ; 217 ; 218 ; 219 ; 260 ; 261 ; 290 ; 291$ \\
\hline 9 & E. floribundus (SDC) & 15,2 & $200 ; 230 ; 311$ \\
\hline 10 & E. floribundus (BTM, ITC) & $15,9-16,2$ & 201; 215; 216; 271; 331; 336 \\
\hline 11 & E. floribundus (BTM, CNM, CPL, CTL, ITC), E. subalatus (MC1, MC2, MC4, SJP) & $16,1-16,9$ & 198; 218; 219; 236; 327; 328 \\
\hline 12 & E. floribundus (BTM, CPL, CTL, ITC, SDC), E. subalatus (MC2, MC4, SJP) & $16,5-17,5$ & $204 ; 229 ; 230 ; 279 ; 280 ; 309 ; 310$ \\
\hline 13 & E. floribundus (BTM) & 17,4 & $204 ; 270 ; 331$ \\
\hline 14 & E. floribundus (CNM) & 17,5 & $231 ; 280 ; 308$ \\
\hline 19 & E. floribundus (ITC) & 20,2 & $200 ; 214 ; 270 ; 336$ \\
\hline 20 & E. floribundus (ITC) & 20,5 & $199 ; 214 ; 270 ; 337$ \\
\hline 21 & E. floribundus (BTM) & 21,3 & $201 ; 327$ \\
\hline 22 & E. floribundus (BTM) & 22,7 & $198 ; 218 ; 235 ; 330$ \\
\hline 23 & E. floribundus (BTM) & 26,5 & $218 ; 236 ; 328$ \\
\hline 24 & E. floribundus (BTM, CNM, CPL, CTL, ITC), E. subalatus (MC1, MC2, MC4, SJP) & $28,7-29,1$ & $217 ; 219 ; 235 ; 236 ; 237 ; 238 ; 329$ \\
\hline 25 & E. subalatus (MC1) & 31,8 & $235 ; 329$ \\
\hline
\end{tabular}

TR: tempo de retenção; $\lambda$ : bandas de absorção no UV-Vis. Números em negrito correspondem às bandas de absorção comuns entre os acessos. CNM: Cônego Marinho; CPL: Capelinha; CTL: Cristália; ITC: Itacambira; MC1: Montes Claros; MC2: Montes Claros; MC4: Montes Claros; SJP: São João da Ponte; STR: Santana do Riacho 


\subsection{Derivados do ácido o-hidroxicinâmico}

O teor detectado na amostra comercial foi inferior ao teor mínimo de derivados do ácido ohidroxicinâmico em droga vegetal de chapéu-de-couro recomendado pela Farmacopeia Brasileira, 2,8\% expressos em verbacosídeos [20]. Já as espécies E. floribundus e E. subalatus apresentaram valores superiores ao determinado pela Farmacopeia Brasileira, 3,07\% e 5,52\% de derivado do ácido hidroxicinâmico expressos em verbacosídeos, respectivamente (Tabela 3). O teor de derivados do ácido hidroxicinâmico em $E$. subalatus é análogo aos valores descritos para a espécie E. grandiflorus por Lopes et al. (2012) [27] e Dias et al. (2013) [18]. Já o teor de derivados do ácido hidroxicinâmico em E. floribundus é superior aos teores descritos em literatura para $E$. grandiflorus (Tabela 3). Esses resultados são indícios de que as espécies estudadas no presente trabalho, $E$. floribundus e E. subalatus, também podem apresentar propriedades medicinais semelhantes às de E. grandiflorus. Conquanto não haja estudos publicados que confirmem, espécies de Echinodorus ecologicamente congêneres aparentam possuir respostas semelhantes [49], justificando, em parte, o uso popular das espécies do presente estudo.

Tabela 3: Determinação do teor de derivados do ácido hidroxicinâmico em amostras de chapéu-de-couro.

\begin{tabular}{ccc}
\hline \multirow{2}{*}{ Espécie } & \multicolumn{2}{c}{ Teor médio de derivados do ácido hidroxinâmico (\%) } \\
\cline { 2 - 3 } & Experimento & Literatura \\
\hline E. floribundus & $5,52 \pm 0,22$ & - \\
E. subalatus & $3,07 \pm 0,15$ & - \\
Amostra comercial & $0,51 \pm 0,74$ & - \\
E. grandiflorus & - & $3,12-3,73[27]$ \\
& - & $2,10-3,89[18]$ \\
\hline
\end{tabular}

\section{CONCLUSÃO}

Desse modo, apesar dos flavonoides, fenóis e derivados $o$-hidroxicinâmico serem reconhecidas como marcadores químicos para Echinodorus floribundus e Echinodorus subalatus a isoorientina e o ácido chicórico foram ausentes, o que as diferencia quimicamente das descritas pela Farmacopeia Brasileira. No entanto, os teores de derivados $o$-hidroxicinâmico indicam potencial farmacológico das espécies.

\section{AGRADECIMENTOS}

O presente trabalho foi realizado com apoio da Coordenação de Aperfeiçoamento de Pessoal de Nível Superior- Brasil (CAPES) - Código de Financiamento 001. À FAPEMIG pelo apoio financeiro ao projeto.

\section{REFERÊNCIAS BIBLIOGRÁFICAS}

1. Matias LQ, Soares G. Morphology and micromorphology of the seed coats of species of Echinodorus (Alismataceae) from Brazilian North-eastern. Bol Museu Paraense Emílio Goeldi, Cienc Nat. 2009 MayAug;4(2):165-173.

2. Tabosa AB, Matias LQ, Martins FR. Live fast and die young: The aquatic macrophyte dynamics in a temporary pool in the Brazilian semiarid region. Aquat Bot. 2012 Oct;102:71-78, doi: 10.1016/j.aquabot.2012.05.004.

3. Lehtonen S. An integrative approach to species delimitation in Echinodorus (Alismataceae) and the description of two new species. Kew Bull. 2008 Dec;63(4):525-563, doi: 10.1007/s12225-008-9068-0.

4. Silva CJ, Bastos JK, Takahashi CS. Evaluation of the genotoxic and cytotoxic effects of crude extracts of Cordia ecalyculata and Echinodorus grandiflorus. J Ethnopharmacol. 2010 Oct;127(2):445-450, doi: 10.1016/j.jep.2009.10.015. 
5. Moro MF, De Souza DJL, Matias LQ. Rarefaction, richness estimation and extrapolation methods in the evaluation of unseen plant diversity in aquatic ecosystems. Aquat Bot. 2014 Jul;117:48-55, doi: 10.1016/j.aquabot.2015.04.006.

6. Oliveira A, Bove C. Checklist of aquatic and marshy Monocotyledons from the Araguaia River basin, Brazilian Cerrado. Biodivers Data J. 2016 Mar;(4):e7085, doi: 10.3897/BDJ.4.e7085.

7. Lima-Dellamora EC, Waldhelm KCV, Alves AM, Lage CAS, Leitão ACC, Kurster RM. Genotoxic Maillard by products in current phytopharmaceutical preparations of Echinodorus grandiflorus. An Acad Bras Cien. 2014 Jan;86(3):1385-1394, doi: 10.1590/0001-3765201420130065.

8. Associação Brasileira das Indústrias de Refrigerantes e de Bebidas Não Alcoólicas. Mate Couro [Internet]; 2015 [acesso em 10 out 2018]. Disponível em: https://abir.org.br/associado/mate-couro/

9. Monteiro ADP. Produção de cervejas especiais adicionadas de extratos solúveis de ervas nativas com propriedades antioxidantes [dissertação]. Curitiba (PR): Universidade Federal do Paraná; 2016.72 p.

10. Brunel S. Pathway analysis: aquatic plants imported in 10 EPPO countries. EPPO bulletin. 2009 Jul;39(2):201-213, doi: 10.1111/j.1365-2338.2009.02291.

11. Ying L, Lei L, PingCheng Y, HuiCai G, XiaoFei H, YongSheng G. A comparison of decontaminating effects of hydroponic plants on domestic sewage. Acta Agric Univ Jiangxiensis. 2014;36(4):881-886.

12. Wan-Ru L, Zhang L, Wei-wei Y. Effect of organic acids amendment on cadmium uptake and translocation by Echinodorus osiris. Turang Tongbao. 2014;45(1):205-209.

13. Barbosa UA, Dos Santos IF, Dos Santos AMP, Dos Santos DC, Da Costa GM. Determination and evaluation of metals and metalloids in the Chapéu-de-couro (Echinodorus macrophyllus (Kunth) Micheli). Biol Trace Elem Res. 2013 Jul;154(3):412-417, doi: 10.1007/s12011-013-9730-8.

14. Ribeiro ES, Pereira MP, De Castro EM, Baroni GR, Corrêa FF, Pereira FJ. Relações da anatomia radicular na absorção, no acúmulo e na tolerância ao chumbo em Echinodorus grandiflorus. Rev Bras Eng Agric Ambient. 2015 May;19(6):605-612, doi: 10.1590/1807-1929/amgriamb.v19n6p605-612.

15. Hasbi M, Budijono B, Hendrizali, A. Heavy metal uptake capacity by floating plant island in sail river pekanbaru. IOP Conf Ser: Earth Environ Sci. 2020;43(1):012035, doi: 10.1088/1755-1315/430/1/012035.

16. Portella VG, Cosenza GP, Diniz LRL, Pacheco LF, Cassali GD, Caliari MV, Brandão MGL, Vieira MAR. Nephroprotective effect of Echinodorus macrophyllus Micheli on gentamicin-induced nephrotoxicity in rats. Nephron Extra. 2012 Jun;2(1):177-183, doi: 10.1159/000339181.

17. Bolson M, Hafler SR, Chaves EID, Gasparotto Jr. A, Cardozo Jr. EL. Ethno-medicinal study of plants used for treatment of human aliments, with residents of the surrounding region of forest fragments of Paraná, Brazil. J Ethnopharmacol. 2015 Feb;161(1):1-10, doi: 10.1016/j.jep.2014.11.045.

18. Dias EGE, Valenzuela VCT, Alves MR, Duarte MGR, Garcia EF. Qualidade e autenticidade de folhas de chapéu-de-couro (Echinodorus grandiflorus) oriundas de fornecedores de São Paulo. Rev Bras Pl Med. 2013 Nov;15(2):250-256, doi: 10.1590/S1516-05722013000200013.

19. Brasil. Farmacopeia Brasileira. 5 ed. Brasília (DF): Agência Nacional de Vigilância Sanitária; 2010. 546 p.

20. Brasil. Farmacopeia Brasileira, Monografias: Plantas Medicinais. 6 ed. Brasília (DF): Agência Nacional de Vigilância Sanitária; 2019. 739 p.

21. Prando TBL, Barboza LN, Araújo VO, Gasparotto FM, De Souza LM, Lourenço ELB, Gasparotto Jr. A. Involvement of bradykinin B2 and muscarinic receptors in the prolonged diuretic and antihypertensive properties of Echinodorus grandiflorus (Cham. \& Scheldt.) Micheli. Phytomedicine. 2016 Jan;23(11):1249-1258, doi: 10.1016/j.phymed.2015.10.020.

22. Garcia EF, De Oliveira MA, Candido LCM, Dourado LPA, De Souza DG, Teixeira MM, Braga FC. In vitro TNF- $\alpha$ inhibition elicited by extracts from Echinodorus grandiflorus leaves and correlation with their phytochemical composition. Planta Med. 2015 Oct;82(04):337-343, doi: 10.1055/ s-0035-1558290.

23. Garcia EF, De Oliveira MA, Candido LCM, Coelho FM, Costa VV, Queiroz-Jr. CM, Boff D, Amaral FA, De Souza DG, Teixeira MM, Braga FC. Effect of the hydroethanolic extract from Echinodorus grandiflorus leaves and fraction enriched in flavone-C-glycosides on antigen-induced arthritis in mice. Planta Med. 2015 Nov;82(5):407-413, doi: 10.1055/s-0035-1568200.

24. Silva GP, Fernandes DC, Vigliano MV, Da Fonseca EN, Santos SVM, Marques PR, Justo MG, Sabino KCC, Coelho MGP. Flavonoid-enriched fraction from Echinodorus macrophyllus aqueous extract exhibits high in-vitro and in-vivo anti-inflammatory activity. J Pharma Pharmacol. 2016 Jul;63(11):15761579, doi: $10.1111 /$ jphp. 12620 .

25. Kobayashi J, Sekiguchi M, Shimamoto S, Shigemori H, Ohsaki A. Echinophyllins C-F, new nitrogencontaining clerodane diterpenoids from Echinodorus macrophyllus. J Nat Prod. 2000 Jul;63(11):15761579, doi: 10.1021/np000355w.

26. Pimenta DS, Figueiredo MR, Kaplan MAC. Essential oil from two populations of Echinodorus grandiflorus (Cham. \& Schltdl.) Micheli (Chapéu de couro). An Acad Bras Cien. 2006 Jul;78(4):623628, doi: 10.1590/S0001-37652006000400002. 
27. Lopes CG, Santos PVP, Diciaula MC, Blainski A, Gutierre MAM, Mello JCP. Validação de metodologia analítica para a determinação de derivados do ácido o-hidroxicinâmico de Echinodorus grandiflorus. Rev Bras Pl Med. 2012;14(3):500-505, doi: 10.1590/S1516-05722012000300012.

28. Pereira HV, Reis FAG, Machado AMR, Marques AR, Garcia CF, Gomes FCO, Nelson DL. Characterization of the volatile compounds and anatomical features in commercial samples of Echinodorus plant species. Research, Society and Development. 2020 Oct;9(10):e8719109199, doi: https://doi.org/10.33448/rsd-v9i10.9199.

29. Tanus-Rangel E, Santos SR, Lima JCS, Lopes L, Noldin V, Monache FD, Cechinel-Filho V, Martins DTO. Topical and systemic anti-inflammatory effects of Echinodorus macrophyllus (Kunth) Micheli (Alismataceae). J Med Food. 2010 Oct;13(5):1161-1166, doi: 10.1089/jmf.2009.0247.

30. Strada CL, Lima KC, Da Silva VC, Ribeiro RV, Dores EFGC, Dall'Oglio EL, Schmeda-Hirschmann G, Carollo CA, Martins DP, De Souza Júnior PT. Isovitexin as marker and bioactive compound in the antinociceptive activity of the Brazilian crude drug extracts of Echinodorus scaber and E. grandiflorus. Rev Bras Farmacogn. 2017 Sept-Oct;27(5):619-626, doi: 10.1016/j.bjp.2017.05.011.

31. Lehtonen S, Myllys L. Cladistic analysis of Echinodorus (Alismataceae): simultaneous analysis of molecular and morphological data. Cladistics. 2008 Oct;24(2):218-239, doi: 10.1111/j.10960031.2007.00177.x.

32. Haynes RR, Holm-Nielsen LB. Notes on Echinodorus (Alismataceae). Brittonia. 1986 Oct;38(4):325332, doi: $10.2307 / 22807073$.

33. Hayness RR, Holm-Nielsen LB. The Alismataceae. Flora Neotropica. 1994 Jul;64(1):1-112.

34. Rataj K. Echinodorus grandiflorus (Cham. et Schltdl.) Mich., its geographical distribution and variability. Folia Geobot Phytot.1969 Sep;4(3):319-326, doi: 10.1007/BF02854750.

35. Lehtonen S. Phylogenetics of Echinodorus (Alismataceae) based on morphological data. Bot J Linn Soc. 2006 Mar; 150(3): 291-305, doi: 10.1111/j. 10958339.2006.00478.x.

36. Matias LQ. O gênero Echinodorus (ALISMATACEAE) do domínio da Caatinga brasileira. Rodriguésia. 2007 Apr;58(4):743-774, doi: 10.1590/2175-7860200758403.

37. Lopes OD, Kobayashi MK, Oliveira FG, Alvarenga IC, Martins ER, Corsato CE. Determinação do coeficiente de cultura $(\mathrm{Kc})$ e eficiência do uso de água do alecrim-pimenta irrigado. Ver Bras Eng Agric Ambient. 2011 Jun;15(6):548-553, doi: 10.1590/S1415-43661011000600002.

38. IBGE (Instituto Brasileiro de Geografia e Estatística). Mapas interativos [Internet]; 2012 [acesso em 15 jan 2018]. Available from: https://mapas.ibge.gov.br/tematicos

39. Silva FA, Bizerra AMC, Fernandes PRD. Testes fitoquímicos em extratos orgânicos de Brixa orellana L (urucum). Holos. 2018 May;2(1):484-498, doi: 10.15628/holos.2018.6929.

40. Matos FJA. Introdução à fitoquímica experimental. 2. ed. Fortaleza (CE): Edições UFC; 1997. 141 p.

41. Amorim ELC, Nascimento JE, Monteiro JM, Peixoto Sobrinho TSJ, Araújo TA, Albuquerque UP. A simple and accurate procedure for the determination of tannin and flavonoid levels and some applications in ethnobotany and ethnopharmacology. Funct Ecosystems Communit. 2008 Oct;2(1):88-94.

42. Peixoto Sobrinho TJS, Da Silva CHTP, Do Nascimento JE, Monteiro JM, De Albuquerque UP, Amorim ELC. Validação de metodologia espectrofotométrica para quantificação dos flavonoides de Bauhinia cheilantha (Bongard) Steudel. Rev Bras Cienc Farm. 2008 Oct-Dec;44(4):683-689, doi: 10.1590/S151693322008000400015 .

43. Garcia EF, De Oliveira MA, Godin AM, Ferreira WC, Bastos LFS, Coelho MM, Braga FC. Antiedematogenic activity and phytochemical composition of preparations from Echinodorus grandiflorus leaves. Phytomedicine. 2010 Dec;18(1):80-86, doi: 10.1016/j.phymed.2010.05.008.

44. Team RC. R: A language and environment for statistical computing. Vienna (AT): R Found Stat Compt; 2014. Disponível em: https://www.R-project.org/

45. Março PH, Poppi RJ, Scarminio IS. Procedimentos analíticos para identificação de antocianinas presentes em extratos naturais. Quím Nova. 2008 Aug;31(5):1218-1223, doi: 10.1590/S0100-40422008000500051.

46. Flor RV, Campos MAA, Solano AGR, Jokl L, Dantas-Barros AM. Drying of Echinodorus macrophyllus and autoclaving and lyophilization of the fluid-extract: effects on the pharmacochemical composition. Rev Bras Farmacogn. 2011 May-Jun;21(3):518-524, doi: 10.1590/S0102-695X2011005000075.

47. Rosa SIG, Rios-Santos F, Balugon SO, De Almeida DAT, Damazo AS, Da Cruz TCD, Pavan E, Barbosa RS, Alvim TC, Soares IM, Ascênio DS, Machado A, Martins DTO. Hydroethanolic extract from Echinodorus scaber Rataj leaves inhibits inflammation in ovalbumin-induced allergic asthma. J Ethnopharmacol. 2017 May;203(1):191-199, doi: 10.1016/j.jep.2017.03.025.

48. Fernández MC, Rubio OC, Hernández IM. Introducción a la caracterización estructural de flavonoides. Machala (CE): UTMACH; 2015. 79 p.

49. Lehtonen S. Natural History of Echinodorus (Alismataceae). Turku (FI): Turun Yliopisto; 2007. 58 p. 